\title{
Pressure ulcers and incontinence-associated dermatitis: effectiveness of the Pressure Ulcer Classification education tool on classification by nurses
}

\author{
Dimitri Beeckman, ${ }^{1,2}$ Lisette Schoonhoven, ${ }^{3}$ Jacqui Fletcher, ${ }^{4}$ Katia Furtado, ${ }^{5}$ \\ Hilde Heyman, ${ }^{6,7}$ Louis Paquay, ${ }^{8,9}$ Dirk De Bacquer, ${ }^{1}$ Tom Defloor $^{1}$
}

${ }^{1}$ Department of Public Health, Ghent University, Gent, Belgium ${ }^{2}$ Department of Bachelor in Nursing, University College Arteveldehogeschool Ghent, Gent, Belgium

${ }^{3}$ IO Scientific Institute for Quality of Healthcare, Radboud University Nijmegen Medical Centre, Nijmegen, The Netherlands

${ }^{4}$ School of Nursing and Midwifery, University of Hertfordshire, Hatfield, UK

${ }^{5}$ Centro de Saude de Arronches, Portalegre, Portugal

${ }^{6}$ Nursing Home Sint Bartholomeus, Antwerp, Belgium

${ }^{7}$ Wound care Consultant Society (WCS), Belgium

${ }^{8}$ Wit-Gele Kruis van Vlaanderen, Brussels, Belgium

${ }^{9}$ Academic Centre for General Practice, KULeuven, Leuven, Belgium

\section{Correspondence to}

Mr Dimitri Beeckman, Nursing Science, Ghent University, UZ Block A 2nd Floor, De Pintelaan 185, Gent B-9000, Belgium; dimitri.beeckman@ugent.be

Accepted 6 March 2009 Published Online First 29 July 2010

\section{ABSTRACT}

Context Previous studies report that pressure ulcer classification and differentiation from incontinence associated dermatitis are difficult. Incorrect classification and differentiation result in incorrect prevention and treatment. Education is important to spread evidencebased insights about this topic and to improve classification skills.

Aim To assess the effectiveness of the Pressure Ulcer Classification (PUCLAS) education tool. PUCLAS was developed by the PUCLAS Workgroup of the European Pressure Ulcer Advisory Panel.

Design Randomised controlled trial.

Setting and participants A convenience sample of 1217 Belgian, Dutch, British and Portuguese nurses.

Outcome measure Correct classification of pressure ulcer photographs and differentiation from photographs of incontinence-associated dermatitis.

Results Baseline, $44.5 \%$ of the photographs were classified correctly. In the post-test, the results in the intervention group were significantly higher (63.2\%) compared with the control group (53.1\%; $p<0.001)$. The percentage of correct assessments of incontinence associated dermatitis (IAD) was $70.7 \%$ in the intervention group and $35.6 \%$ in the control group $(p<0.001)$. The skill to differentiate IAD from pressure ulcers was significantly associated with the experimental intervention (OR 4.07, 95\% Cl 3.21 to $5.15, \mathrm{p}<0.001$ ). Conclusion The PUCLAS tool improved pressure ulcer classification and IAD differentiation significantly.

\section{INTRODUCTION}

Pressure ulcers are complex lesions of the skin and underlying structures caused by prolonged pressure on the tissues or by shearing forces. ${ }^{1}{ }^{2}$ Pressure ulcers are a major burden in terms of patient suffering, mortality, morbidity, rehabilitation and healthcare expenditures. ${ }^{3-6}$ Decreased health and restricted mobility make hospitalised patients and nursing home residents particularly vulnerable. ${ }^{7}$

Pressure ulcers are an internationally recognised patient safety problem and are often preventable. Despite the increasing expenditure on prevention, pressure ulcers remain a major healthcare problem. ${ }^{8-10}$ In 2002, prevalence in European hospitals was $18.1 \%$. Only $10 \%$ of the patients in need of prevention received adequate prevention. ${ }^{9}$ Pressure ulcer prevention is a responsibility of all healthcare professionals involved in patient care. ${ }^{11}$
Classification systems are accepted standards to determine pressure ulcer severity. ${ }^{12}$ Recent studies showed that pressure ulcer classification is difficult ${ }^{13-18}$ and that misclassification between pressure ulcers and incontinence-associated dermatitis (IAD) frequently occurs. ${ }^{14}{ }^{15}{ }^{19}$ IAD is an inflammation of the skin that occurs when urine or faeces comes into contact with perineal or perigenital skin. The lesions are characterised by erosion of the epidermis and a macerated appearance of the skin. ${ }^{20}$ Differential diagnosis between pressure ulcers and IAD is based on visual examination. ${ }^{21-23}$ Misclassification has significant implications for prevention, treatment, and reporting and benchmarking on quality of care.

Classification skills are likely to benefit from education. No research reporting the effectiveness of education on classification skills could be found. This study aims to evaluate the effectiveness of the Pressure Ulcer Classification (PUCLAS) education tool on classification skills by nurses.

\section{METHODS}

\section{Study design and study population}

A randomised controlled trial design was used. A convenience sample of 1217 nurses from Belgium, The Netherlands, UK and Portugal participated. Data were collected between September 2005 and December 2006. Nurses attending a wound care conference were approached to participate in the study. Before data collection, the purpose and procedure were explained, and anonymity and confidentiality were ensured. The study was approved by the ethics review committee of Ghent University Hospital. The ethics committee waived the need for written informed consent. A completed questionnaire was taken as consent to participate.

\section{Operational definitions}

European Pressure Ulcer Advisory Panel classification system

The European Pressure Ulcer Advisory Panel (EPUAP) classifies pressure ulcers in four grades based on the severity of the lesion. This European classification system is widely used for application in research, teaching and patient care (table 1). ${ }^{24}$

PUCLAS education tool

PUCLAS is a tool to teach and learn about pressure ulcer classification and IAD differentiation, and was developed by the PUCLAS Workgroup of the 
Table 1 European Pressure Ulcer Advisory Panel classification system $^{24}$

Grade 1 Non-blanchable erythema of intact skin. Discoloration of the skin, warmth, oedema, induration or hardness may also be used as indicators, particularly in individuals with darker skin

Grade 2 Partial thickness skin loss involving epidermis, dermis, or both. The ulcer is superficial and presents clinically as an abrasion or blister

Grade 3 Full thickness skin loss involving damage to or necrosis of subcutaneous tissue that may extend down to, but not through, underlying fascia

Grade 4 Extensive destruction, tissue necrosis, or damage to muscle, bone, or supporting structures with or without full thickness skin loss

EPUAP. It is based on the EPUAP position statement on pressure ulcer classification and IAD differentiation, and provides an overview of causative factors and of typical wound-related characteristics including location, shape, depth, necrosis, edges and colour (table 2). ${ }^{22} 23$

\section{Data collection}

Photographs were used for the assessments. Prior to this study, two sets of 20 photographs were validated. Every set consisted of one photograph of normal skin, one photograph of blanchable erythema, three photographs of each pressure ulcer grade, three photographs of IAD and three photographs of a combination of a pressure ulcer and IAD. The sets were validated in a double Delphi procedure by 12 trustees of EPUAP who have an extensive experience in pressure ulcer research. All experts adopted the classification of the photographs unanimously. This experts' opinion was considered the gold standard.

To gain baseline information on classification skills, the nurses classified one set of 20 photographs. The photographs were projected on a white background. The participants received no feedback on their assessments. Subsequently, participants were randomly assigned to an intervention and a control group by means of a computer-generated sequence of random numbers. The intervention group attended the PUCLAS education in a private classroom. This intervention consisted of a standardised $1 \mathrm{~h}$ face-to-face lecture. In the PUCLAS intervention, clinical differences between pressure ulcers and IAD were presented by means of definitions, photographs and video. Exercises on different levels of difficulty were included (http:// www.puclas.ugent.be). The lecturers were member of the PUCLAS Workgroup of EPUAP and had comprehensive experience in pressure ulcer care. The lecture was illustrated with PowerPoint $^{\circledR} 2003$ (Microsoft Corporation ${ }^{\circledR}$, Redmond, WA, USA). The control group received a $15 \mathrm{~min}$ standardised rehearsal of the definitions of the pressure ulcer grades within the EPUAP classification system. No time elapsed between the intervention and post-test. During the post-test, the participants classified both sets of photographs, that is, 40 photographs. A scoring form, formatted with the software package Teleform ${ }^{\circledR}$ (Cardiff $^{\circledR}$, Vista, CA, USA), was used for the assessment.

\section{Data analysis}

As each nurse rated a fixed set of pressure ulcer photographs, differences in classification skills between experimental and control group were analysed according to multilevel logistic modelling reflecting the hierarchical clustering of photographs within nurses. In these models, potential confounding due to differences in distributions of country, educational level and work experience was adjusted for. The estimated effect of the intervention was expressed according to OR (95\% CI). All statistical analyses were performed using SAS software release 9.1.3 (SAS Institute, Cary, North Carolina). The Glimmix Procedure was used. A level of $\alpha=0.05$ was chosen a priori to indicate statistical significance.

\section{RESULTS}

\section{Baseline characteristics}

A total of 1217 nurses from Belgium (45.0\%; $n=548$ ), The Netherlands $(33.7 \% ; n=410)$, UK $(17.4 \% ; n=212)$ and Portugal $(3.9 \% ; n=47)$ participated in this study. About $70 \%$ of the study nurses were between 20 and 45 years old, and $30.0 \%$ were over the age of 45 years. Approximately $70 \%$ had more than 10 years of experience, and $30.3 \%$ worked as a nurse for more than 20 years. All participants stated that they were familiar with the use of the EPUAP classification scale (table 3).

Baseline, 44.5\% (10498/23595) of the photographs were classified correctly. There was no statistical difference between the results of the intervention group (44.6\%) and the control group (44.3\%; $\mathrm{p}=0.82$; table 4).

\section{Effectiveness of the PUCLAS education}

After the intervention, the participants in the intervention group were consistently more likely to classify correctly IAD photographs (OR 4.07, 95\% CI 3.21 to $5.15, \mathrm{p}<0.001$ ) and photographs of a combination of a pressure ulcer and IAD (OR $2.08,95 \%$ CI 1.73 to $2.50, \mathrm{p}<0.001$ ) when classifying the new set of photographs as a result of the experimental intervention. Similar results were found when classifying the same photographs from the pretest. There was no difference between the intervention group and the control group for the classifications of grade 1 and grade 4 pressure ulcers (table 5). The percentage of correct assessments of IAD was $70.7 \%$ in the intervention group and $35.6 \%$ in the control group $(p<0.001)$.

Table 2 Synthesis of the European Pressure Ulcer Advisory Panel position statement on pressure ulcer classification and incontinence-associated dermatitis (IAD) differentiation ${ }^{22} 23$

\begin{tabular}{|c|c|c|}
\hline & Pressure ulcer & IAD \\
\hline Cause & Pressure and/or shear must be present & $\begin{array}{l}\text { Moisture must be present (eg, shining, wet skin caused by urinary } \\
\text { incontinence or diarrhoea) }\end{array}$ \\
\hline Location & A wound over a bony prominence is likely to be a pressure ulcer & $\begin{array}{l}\text { IAD may occur over a bony prominence; however, pressure and shear } \\
\text { should be excluded as causes, and moisture should be present }\end{array}$ \\
\hline Shape & If the lesion is limited to one spot, it is likely to be a pressure ulcer & Diffuse, different superficial spots are more likely to be IAD \\
\hline Depth & Partial thickness skin loss and full thickness skin loss & Superficial (partial thickness skin loss) \\
\hline Necrosis & $\begin{array}{l}\text { A black necrotic scab on a bony prominence is a pressure ulcer grade } \\
3 \text { or } 4 \text {; if there is no or limited muscular mass underlying the necrosis, } \\
\text { the lesion is a pressure ulcer grade } 4\end{array}$ & No necrosis \\
\hline Edges & Distinct edges & Diffuse or irregular edges \\
\hline Colour & If redness is non-blanchable, this is most likely a pressure ulcer grade 1 & $\begin{array}{l}\text { Blanchable or non-blanchable erythema; pink or white surrounding skin due } \\
\text { to maceration }\end{array}$ \\
\hline
\end{tabular}


Table 3 Characteristics of study nurses

\begin{tabular}{lcc}
\hline & $\begin{array}{l}\text { Intervention }(\mathbf{n}=658), \\
\text { percentage (n) }\end{array}$ & $\begin{array}{c}\text { Control }(\mathbf{n}=559) \\
\text { percentage (n) }\end{array}$ \\
\hline Gender (percentage male) & $13.5(87 / 643)$ & $12.2(68 / 558)$ \\
Expertise (percentage expert) & $35.1(224 / 639)$ & $30.0(163 / 544)$ \\
Education & & \\
$\quad$ Undergraduate & $45.7(300 / 655)$ & $58.3(326 / 559)$ \\
$\quad$ Bachelor & $48.9(321 / 655)$ & $36.5(204 / 559)$ \\
$\quad$ Master & $5.2(34 / 655)$ & $5.2(29 / 559)$ \\
Age category (years) & & \\
$<25$ & $5.8(38 / 658)$ & $12.2(68 / 559)$ \\
$25-35$ & $27.4(180 / 658)$ & $24.2(135 / 559)$ \\
$35-44$ & $37.1(244 / 658)$ & $32.6(182 / 559)$ \\
$>44$ & $29.8(196 / 658)$ & $31.1(174 / 559)$ \\
Work experience (years) & & \\
$<5$ & $13.1(86 / 658)$ & $18.8(103 / 548)$ \\
$5-10$ & $14.4(95 / 658)$ & $17.5(96 / 548)$ \\
$10-20$ & $40.9(269 / 658)$ & $34.9(191 / 548)$ \\
$>20$ & $31.6(208 / 658)$ & $28.8(158 / 548)$ \\
Country & & \\
Belgium & $60.6(399 / 658)$ & $26.7(149 / 559)$ \\
The Netherlands & $15.3(101 / 658)$ & $55.3(309 / 559)$ \\
Portugal & $4.6(30 / 658)$ & $3.0(17 / 559)$ \\
UK & $19.5(128 / 658)$ & $15.0(84 / 559)$ \\
\hline
\end{tabular}

\section{DISCUSSION}

Before training, the classification skills of the study nurses were poor. Pressure ulcers and IAD were frequently classified incorrectly. After reviewing the EPUAP classification system, limited improvement was seen in the observation of IAD. Attending PUCLAS resulted in significantly more correct observations of $\mathrm{IAD}$ compared with the control group.

Based on this study, we can assume that the description of clinical guidelines about the differential diagnosis between pressure ulcers and IAD provided a positive effect. The effectiveness was probably supported by the use of clear definitions, descriptions and exercises. The theoretical insights were depicted unambiguously and, where suitable, graphically supported.

Ethical, legal and financial implications mean that a correct differentiation between pressure ulcers and IAD is important. Incorrect differentiation results in inadequate preventive and therapeutic measures, and in suboptimal use of available resources. Prevention of IAD comprises a structured skin care regimen including gentle cleansing, moisturisation and application of a skin protectant or moisture barrier. Treatment goals include protecting the skin from further exposure to irritants, establishing a healing environment and eradicating cutaneous infection. ${ }^{2021}$ Contrarily, prevention of pressure ulcers comprises

Table 4 Classification skills of the study nurses in the pretest

\begin{tabular}{llll}
\hline & $\begin{array}{l}\text { Intervention }(\mathbf{n}=658), \\
\text { percentage }(\mathbf{n})\end{array}$ & $\begin{array}{l}\text { Control }(\mathbf{n}=559), \\
\text { percentage }(\mathbf{n})\end{array}$ & Significance* \\
\hline All photographs & $44.6(5703 / 12772)$ & $44.3(4795 / 10823)$ & $\mathrm{p}=0.82$ \\
Normal skin & $95.1(621 / 653)$ & $94.1(514 / 546)$ & $\mathrm{p}=0.19$ \\
Blanchable erythema & $68.8(440 / 640)$ & $68.6(372 / 542)$ & $\mathrm{p}=0.13$ \\
PU grade 1 & $43.8(840 / 1917)$ & $38.2(620 / 1625)$ & $\mathrm{p}=0.17$ \\
PU grade 2 & $45.8(871 / 1901)$ & $52.4(855 / 1630)$ & $\mathrm{p}=0.11$ \\
PU grade 3 & $35.2(672 / 1908)$ & $38.8(624 / 1608)$ & $\mathrm{p}=0.32$ \\
PU grade 4 & $51.8(996 / 1924)$ & $51.7(841 / 1626)$ & $\mathrm{p}=0.62$ \\
IAD & $32.2(617 / 1917)$ & $25.5(413 / 1621)$ & $\mathrm{p}=0.47$ \\
PU/IAD & $33.8(646 / 1912)$ & $34.2(556 / 1625)$ & $\mathrm{p}=0.21$ \\
\hline
\end{tabular}

*Adjusted for country, educational level, work experience and accounting for clustering of photos within nurses.

IAD, incontinence-associated dermatitis; PU, pressure ulcer; PU/IAD, combination of pressure ulcer and incontinence-associated dermatitis. a reduction in the intensity and/or duration of pressure and shearing forces on the tissue. Pressure-relieving mattresses, cushions and postures are some of the possibilities that can be used. $^{25}$

Correct classification of pressure ulcers and differentiation between IAD requires a profound knowledge and clear understanding of the different skin layers, physiology and pathology of the skin. Care givers should be trained prior to undertaking pressure ulcer classification. Tissue viability training and unambiguous observation guidelines are important and should be presented at an appropriate level to maximise retention and to ensure an adequate adoption of skills in daily practice.

PUCLAS is developed for both clinical basic and in-service education. The programme can be used as a stand-alone teaching module, as an e-learning module or as a supporting online educational package for blended learning. PUCLAS can easily be implemented by healthcare organisations (hospitals, nursing homes, home care) to teach staff about pressure ulcer classification and IAD differentiation. It is important to note that this knowledge will probably not be static and that PUCLAS should be regularly presented. More research is needed to evaluate whether better classification skills and skills to differentiate between pressure ulcers and $\mathrm{IAD}$ would improve preventive care of pressure ulcers and $\mathrm{IAD}$.

The identification of non-blanchable erythema seemed to remain difficult. Accurate identification of non-blanchable erythema (grade 1) is important in pressure ulcer prevention. Research shows that non-blanching erythema with or without other skin changes differs from normal skin/blanching erythema and is associated with a higher risk for pressure ulcer development. $^{2627}$ Confusion about a grade 1 pressure ulcer will probably result in a delayed application of preventive measures and subsequently pressure ulcer development. Recent research showed that using non-blanchable erythema as an indicator to start prevention led to a considerable reduction in patients in need of prevention without an increase in pressure ulcers. ${ }^{28}$ In this study, the confusion about non-blanchable erythema might be caused by the use of photographs providing only a twodimensional view of the lesion. As a result, the dynamic process of blanching erythema could not be presented fully.

An alternative for the use of photographs could be skin assessment in clinical practice. Whether skin assessment in clinical practice is easier than using photographs is unknown. Skin assessment in clinical practice will cost more time and will be more small-scale and harder to organise. Ethical issues should also be considered. Photographs, combined with information about the medical and wound history of the patient, his mobility, incontinency status and nutritional condition might be more effective. Cases including photographs or video material could be an alternative and should be a subject for further research.

Pressure ulcer classification improved in both the intervention and the control group, but did not become optimal. After the intervention, pressure ulcer classification remained difficult. Based on this study, we suggest that the complexity of the fourgrade EPUAP classification system should be evaluated. It could be questioned whether it is important to classify pressure ulcers in four grades. Each grade indicates the degree of tissue damage, but provided limited indication (depth) for treatment and evaluation. An alternative could be to switch to a less complex classification system. This new classification should make a distinction between healthy skin, non-blanchable erythema and a pressure ulcer. The distinction between pressure ulcers and $\mathrm{IAD}$ is more important for determining a correct therapy than being able to classify pressure ulcers in different grades. 
Table 5 Classification skills of the study nurses in the post-test

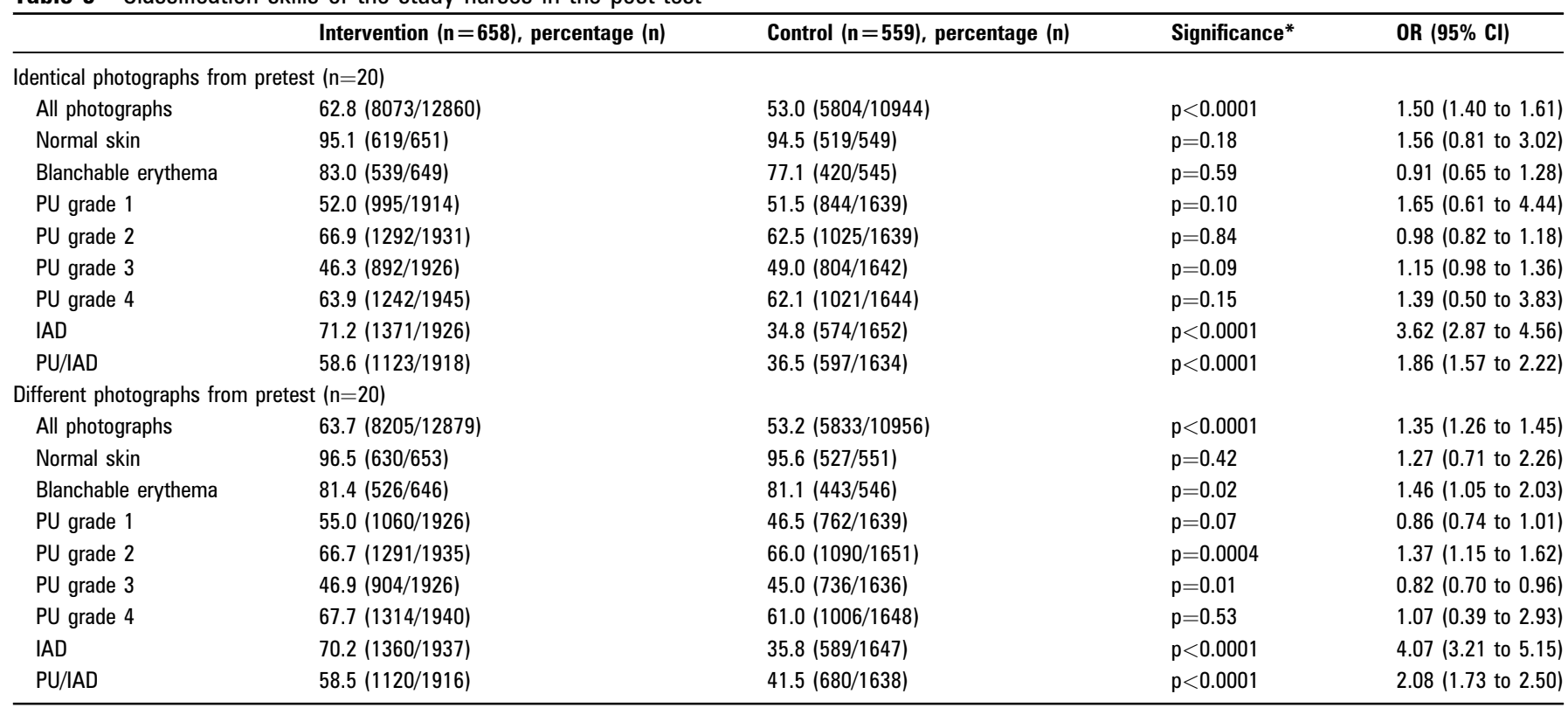

${ }^{*}$ Adjusted for country, educational level, work experience and accounting for clustering of photos within nurses.

$I A D$, incontinence-associated dermatitis; PU, pressure ulcer; PU/IAD, combination of pressure ulcer and incontinence-associated dermatitis.

\section{LIMITATIONS}

The fact that nurses attending a wound care conference were approached to participate in the study could have an effect on the outcomes of this study. The participants were possibly more interested in wound care. Because the nurses all stated that they were familiar with the EPUAP classification system, it is reasonable to assume that the skills at baseline are rather presented too 'positive.'

\section{CONCLUSION}

Attending PUCLAS improved pressure ulcer classification and IAD differentiation significantly. PUCLAS is found to be effective to facilitate learning about this topic. Improved classification skills and ability to differentiate between pressure ulcers and IAD will probably result in more adequate prevention and treatment.

Competing interests None.

Ethics approval Ethics approval was provided by the Ethics Committee of Ghent University Hospital.

Provenance and peer review Not commissioned; externally peer reviewed.

\section{REFERENCES}

1. Defloor T. The risk of pressure ulcers: a conceptual scheme. J Clin Nurs 1999;8:206-16.

2. Panel for the Prediction and Prevention of Pressure Ulcers in Adults. Pressure ulcers in adults: prediction and prevention. Clinical practice guideline 3. Rockville, MD: AHCPR, United States Department of Health and Human Services, Public Health Service (US), 1992. Publication No.: 92-0047.

3. Hopkins A, Dealey C, Bale $\mathrm{S}$, et al. Patient stories of living with a pressure ulcer $J$ Adv Nurs 2006;56:345-53.

4. Allman RM, Goode PS, Burst N, et al. Pressure ulcer, hospital complications, and disease severity: impact on hospital costs and length of stay. Adv Wound Care 1999:12:22-30.

5. Lyder CH, Shannon R, Empleo-Frazier 0, et al. A comprehensive program to prevent pressure ulcers in longterm care: exploring costs and outcomes. Ostomy Wound Manage 2002;48:52-62.

6. Thomas D, Goode PS, Tarquine PH, et al. Hospital-acquired pressure ulcers and risk of death. J Am Geriatr Soc 1996;44:1435-40.

7. Baumgarten M, Margolis D, Localio A, et al. Pressure ulcers among elderly patients early in the hospital stay. J Gerontol A Biol Sci Med Sci 2006;61:749-54.

8. Bergstrom N. Patients at risk for pressure ulcers and evidence-based care for pressure ulcer prevention. In: Bader D, Bouten C, Colin D, et al, eds. Pressure ulcer research. Berlin: Springler-Verlag, 2005:35-50.
9. Vanderwee $\mathbf{K}$, Clark M, Dealey C, et al. Pressure ulcer prevalence in Europe: a pilot study. J Eval Clin Pract 2007;13:227-35.

10. Arnold MC. Pressure ulcer prevention and management: the current evidence for care. AACN Clin Issues 2003:14:411-28.

11. Robinson C, Gloekner M, Bush S, et al. Determining the efficacy of a pressure ulce prevention program by collecting prevalence and incidence data: a unit-based effort. Ostomy Wound Manage 2003;49:44-6, 48-51.

12. Dealey C, Lindholm C. Pressure ulcer classification. In: Romanelli M, Clark M, Colin D, et al, eds. Science and practice of pressure ulcer management. London: European Pressure Ulcer Advisory Panel \& Springler-Verlag, 2006:37-41

13. Bours GJ, Halfens RJ, Lubbers M, et al. The development of a national registration form to measure the prevalence of pressure ulcers in The Netherlands. Ostomy Wound Manage 1999;45:28-33, 36-8, 40

14. Defloor T, Schoonhoven L, Vanderwee K, et al. Reliability of the European Pressure Ulcer Advisory Panel classification system. J Adv Nurs 2006;54:189-98.

15. Defloor T, Schoonhoven L. Inter-rater reliability of the EPUAP pressure ulcer classification system using photographs. J Clin Nurs 2004;13:952-9.

16. Pedley G. Comparison of pressure ulcer grading scales: a study of clinical utility and inter-rater reliability. Int J Nurs Stud 2004:41:129-40.

17. Russell L, Reynolds TM. How accurate are pressure ulcer grades? An image-based survey of nurse performance. J Tissue Viability 2001;11:67, 70-5.

18. Russell L. Pressure ulcer classification: the system and the pitfalls. Br J Nurs 2002:11(12 Suppl):S49-50, S52, S54-7 passim.

19. Weir D. Pressure ulcers: assessment, classification, and management. In: Krasner DL, Rodeheaver GL, Sibbald RG, eds. Chronic wound care: a clinical source book for healthcare professionals. 4th edn. Malevern (PA): HMP Communications, 2007:575-81.

20. Gray M, Bliss DZ, Doughty DB, et al. Incontinence-associated dermatitis: a consensus. J Wound Ostomy Continence Nurs 2007;34:45-54; quiz 55-6.

21. Gray M, Bohacek L, Weir D, et al. Moisture vs pressure: making sense out of perineal wounds. J Wound Ostomy Continence Nurs 2007;34:134-42.

22. European Pressure Ulcer Advisory Panel (EPUAP). Pressure ulcer classification: differentiation between pressure ulcers and moisture lesions. EPUAP Review 2005;6:35-8.

23. Defloor T, Schoonhoven L, Fletcher J, et al. Statement of the European Pressure Ulcer Advisory Panel_-pressure ulcer classification: differentiation between pressure ulcers and moisture lesions. J Wound Ostomy Continence Nurs 2005;32:302-6; discussion 306.

24. EPUAP. Guidelines on treatment of pressure ulcers. EPUAP Review 1999;1:31-3.

25. Defloor T. The effect of position and mattress on interface pressure. Appl Nurs Res 2000;13:2-11.

26. Allman RM, Goode PS, Patrick MM, et al. Pressure ulcer risk factors among hospitalized patients with activity limitation. JAMA 1995;273:865-70.

27. Nixon J, Cranny G, Bond S. Skin alterations of intact skin and risk factors associated with pressure ulcer development in surgical patients: a cohort study. Int J Nurs Stud 2007:44:655-63.

28. Vanderwee K, Grypdonck M, Defloor T. Non-blanchable erythema as an indicator for the need for pressure ulcer prevention: a randomized-controlled trial. J Clin Nurs 2007; 16:325-35 